\title{
Analysis and Stabilization of Image Using Mems Gyroscope
}

\author{
Prince Sachdeva ${ }^{1}$, Paras Chawala ${ }^{2}$ \\ ${ }^{1}$ (M.Tech Scholar, ECE Department, JMIT, Radour, Yamuna Nagar, Haryana, India) \\ ${ }^{2}$ (Professor, ECE Department, CEC, Landran, Greater Mohali, Punjab, India) \\ ( ${ }^{1}$ princesachdeva20@gmail.com, ${ }^{2}$ dr.paraschawla@cgc.edu.in, ${ }^{2}$ pc.monti15@gmail.com)
}

\begin{abstract}
MEMS gyroscope is a very important device used to measure angular rate. Gyroscopes play a vital role when we need the information about the orientation of an object and the motion of current in it. MEMS gyroscopes have a wide applications in defense, navigations, computer industries etc. In this paper the gyroscope has been used in a self designed science vehicle to stabilize the image of the ground from a height of nearly $800 \mathrm{~m}$. This was achieved with the help of 3-servo motors which are attached to each other. Another anti-gyro mechanism was used to control the speed of the Science Vehicle with the help of blades which were tilted in such a way that they were able to reduce the speed of Science Vehicle.The purpose was to simulate the Science Vehicle so that it could travel through a planetary atmosphere sampling the atmospheric composition like outside pressure, temperature, velocity, decent rate etc. during descent.
\end{abstract}

\section{INTRODUCTION}

MEMS is a process technology used to create tiny integrated devices using mechanical and electrical/electronic components. They are fabricated using IC Batch processing techniques and range in size from micrometers to millimeters. They sense, control, actuate on micro scale, and generate readings and information on very precise results at macro level. Electronic devices are fabricated using computer IC Technology, micromechanical components are fabricated by sophisticated manipulations of silicon and other substrates using micro machine processes. Current MEMS devices include accelerometers for air bag sensors, ink jet printer heads, computer disk drive read/write heads, projection display chips, blood pressure sensors, optical switches, microvalves, biosensors and many other products that are all manufactured and shipped in high commercial volumes. If semiconductor micro fabrication was seen to be the first micro manufacturing revolution, MEMS is the second revolution.

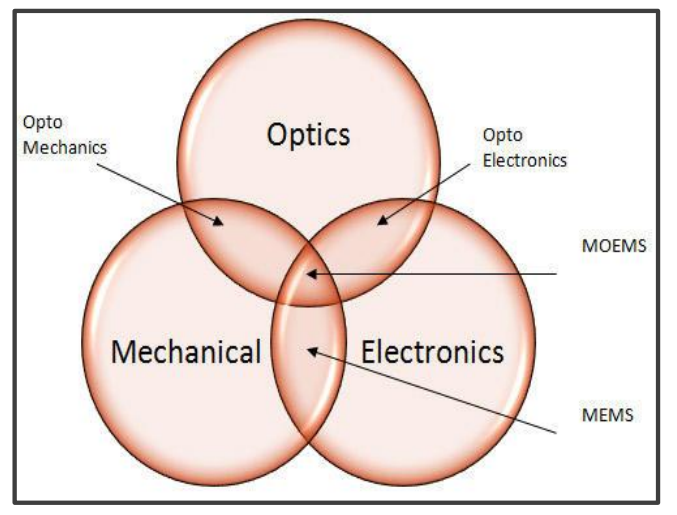

Fig 1: Illustrates the classifications of micro systems technology (MST)

The real difference between MEMS and MST is that MEMS tends to use semiconductor processes to create a mechanical part rather than the deposition of a material on silicon. The most widely used devices in MEMS are transducers, sensors and actuators. MEMS applications are diverse and oldest application is pressure sensors. The other major sensing market is inertial sensors include accelerometers and gyroscopes. MEMS gyroscopes are silicon 
based sensors used to measure angular rate. It is ideal for motion based system, where information about an object's orientation or current motion is important. It can be used to detect how many degree has moved. MEMS gyroscope have been introduced into consumer space to support hand held devices and enabling interesting form of human computer interaction. It is mainly used in image stabilization as unintentional motion can be detected .It relates to equipment's monitoring robotics systems, and platform stabilization. It helps manager to detect when machinery is acting irregularity or hydraulic systems are not performing properly. In industries where large robotics arm used for manufacturing there, MEMS gyroscope determine the orientation of arms at proper angle and it monitor the movement. MEMS gyroscope has roots on aerospace and defense sectors. It measure angular rate changes, they are ideal for unnamed vehicle, missile guidance system, inertial navigation and application.

\section{LITERATURE SURVEY}

[1] Ali Esmaeili, 2013, This article presents a novel method to optimize the sensitivity and robustness of MEMS vibratory gyro-scopes with the help of a 3-DOF MEMS vibratory gyroscope with a 2-DOF-sense mode, which enables adjusting an adaptive trade-off between the precision and robustness for their different desirable importance ratios and a broadband range of frequency response. A comparison is made among a fully coupled gyroscope with no adjustment capability, an adjustable and fully coupled gyroscope, and an adjustable and broadband gyroscope. Then, they are simulated with different weighting factors. Comparing the results shows the superiority of the proposed adjustable as well as broadband gyroscope.

[2] Tao Jiang, 2006, the detection capacitance analysis method based on the elastic body model for the tuning fork vibratory micro machined gyroscope is first put forward to improve the analytical precision of detection capacitance by employing a dynamic finite element. In this way, the simulation of the microgyroscope performance variance due to its structural variance inevitably introduced by the process of practical micromachining can be achieved.

[3] Wei Wang, 2014, this paper presents a novel multi-degree-of-freedom (multi-DOF) micro machined vibratory gyroscope design operated at atmospheric pressure. In this design, the complete 2-DOF vibratory structure is utilized in drive-mode and sense-mode and also, the 2-DOF sense-mode is implemented in both driving frame and proof frame, which form the double 2-DOF sense-modes.

[4] Sang Won Yoon, 2012, this paper analyzes potential causes of vibration-induced error in ideal MEMS tuning fork gyroscopes. Even though MEMS gyros are known to be highly susceptible to mechanical vibration, the mechanisms responsible for generating vibration-induced errors are not well understood. We focus on the tuning fork gyroscope (TFG) design that is known to be relatively immune to vibration because of its differential operation and common-mode rejection.[5] Arashk Norouzpur-Shirazi, 2014, This paper introduces a digital phase demodulation technique for resonant MEMS gyroscopes. The proposed method converts the amplitude-modulated Coriolis signal of the gyroscope into a digital phase-modulated output by utilizing the quadrature component of the sense signal. The rate information is extracted from the digital phase-modulated output using an XOR gate as a digital multiplier. A scale factor of $240 \mathrm{mV} / \% \mathrm{~s}$ with sub $-0.001 \% \mathrm{~s}$ detectable rate is measured. The complete system exhibits a low bias instability of $0.55 \% \mathrm{~h}$ and an angle random walk of $0.12 \% / \mathrm{h}$.

[6] Hung Ngoc Vu, 2013, in this paper, we present design and analysis of a three-degree-of-free sensor, which integrates a lateral axis tuning fork angular rate gyroscope with a two-degree-of-free accelerometer. The sensors for integrating are designed to reduce noises and improve the sensitivity by exploring mechanical couple springs.[7] Vithya Saahar, 2013, The Paper focuses about designing a solid state tuning fork gyroscope based on MEMS that gives accuracy and reliability and that can be commercially viable with cost effective. Also this project includes mathematical modeling in which energy and voltage can be calculated.

[8] Sheng-Ren Chiu, 2012, this paper deals with the design, fabrication and preliminary experimental characterizations of a novel integrated dual-axis tuning fork gyroscope (DTFG). The DTFG is fabricated by highaspect-ratio silicon-on-glass (SOG) process and vacuum packaged by glass frit bonding. [9] Heewon Jeong, 2011, a three-axis microelectromechanical systems (MEMS) inertial sensor measuring two-axis acceleration and angular rate (rotation) has been developed for an electronic stability control (ESC) system for automobiles. We combined 
the angular rate detection part with the two-axis acceleration detection parts on a single MEMS chip to miniaturize the sensor. [10] Chun-Wei Tsai, 2012, this study developed a microelectro mechanical systems (MEMS) doubly decoupled gyroscope with a wide driving frequency range. The gyroscope increases the resonance bandwidths of both the drive and sense oscillators without sacrificing their $\mathrm{Q}$ factors. This bandwidth enhancement ensures good frequency matching between the drive and sense oscillators even if fabrication imperfections are present.

\section{MATHEMATICAL CALCULATION}

For the analysis of various upcoming devices related to MEMS technology in almost all fields of work either it's a field of medical science, defense, computer generation, daily work load or in the world of electronics, the study of MEMS technology is looking to be the future of the world, so beyond a part of various MEMS devices, The aim is to study various threats related to a MEMS device that is GYROSCOPE. Due the various reasons, related to its scope in nearly all the devices in which the angular rate is required.

Gyroscope is derived from Greek words " gyro" meaning to view and scope means "to view". It is a sensor that measure rate of rotation of object. It is used for inertial navigation, image stabilization and auto motive classical control $\mathrm{n}$ rollover direction. Gyroscopes are designed to sense rotation, which they do by taking advantage of the Coriolis Effect. The Coriolis Effect is the name given to the acceleration a moving body within a rotating reference frame seems to experience.

The design of a gyroscope is very important for its application. The most common gyroscope is the Micro-Electro Mechanical System (MEMS) tuning for gyroscope. This gyroscope is designed with a symmetrical tuning fork structure. This design uses two proof masses. The structure is composed of long thin beams and anchored in the center, leaving the proof masses free to move. It is essential to adjust all of the dimensions so that the desired frequency range is attained, allowing the data to be collected and interpreted without excess interference from background noise.

MEMS tuning-fork style gyroscopes are small silicon devices that detect rotation. Most gyroscopes are now made from layered silicon wafers. These wafers are micro machined such that the gyroscope's form is exposed from the middle of the wafer. These gyroscopes are basically designed to work in the frequency range of 10 to $20 \mathrm{kHz}$. These systems need to operate in a temperature range of $-40^{\circ} \mathrm{C}$ to $80^{\circ} \mathrm{C}$. The tuning fork gyroscope that we have studied is the single axis gyroscope i.e. is used to sense the rotation only in one direction. One of the major advancement is the MEMS 3 -axis gyroscope that is used to measure the rotation along 3 orthogonal sensitive axes. Figure 2 shows the mechanical structure of gyroscope:

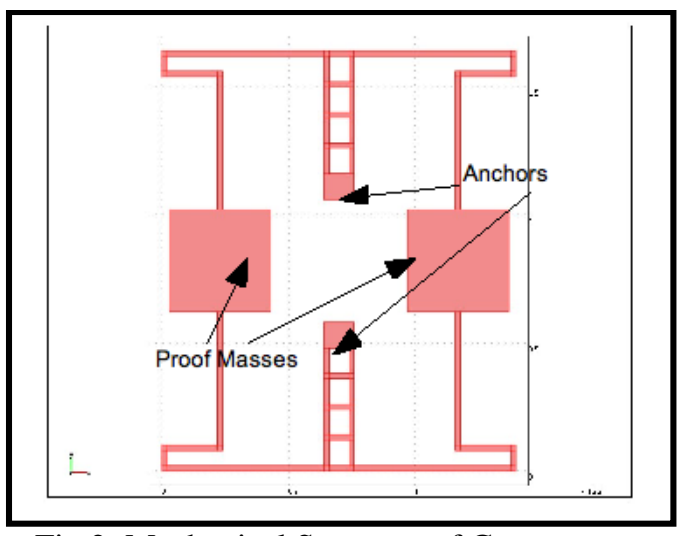

Fig 2: Mechanical Structure of Gyroscope

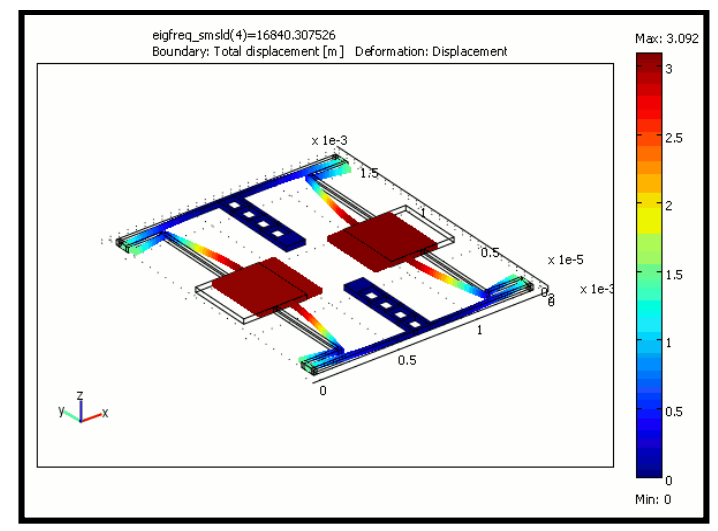

Fig 3: Drive Mode Frequency (motion along x-axis)

The structure is fixed at the anchors shown in the above figure, while the rest of the structure, including the large proof masses on either side, is free to move. Since the gyroscope uses the Coriolis Effect to detect rotation, the device must be in constant motion to function as the Coriolis force only acts on moving bodies. 
This motion is accomplished by vibrating the structure at one of its natural frequencies to achieve the motion shown in Figure 3, this vibration is referred to as the Drive Mode. When the structure begins to rotate, Coriolis force acting on the moving proof masses changes the direction of the vibration from horizontal to vertical as seen in Figure 4. This vertical vibration corresponds to a high natural frequency of the structure than the horizontal Drive Mode vibration and is referred to as Sense Mode.

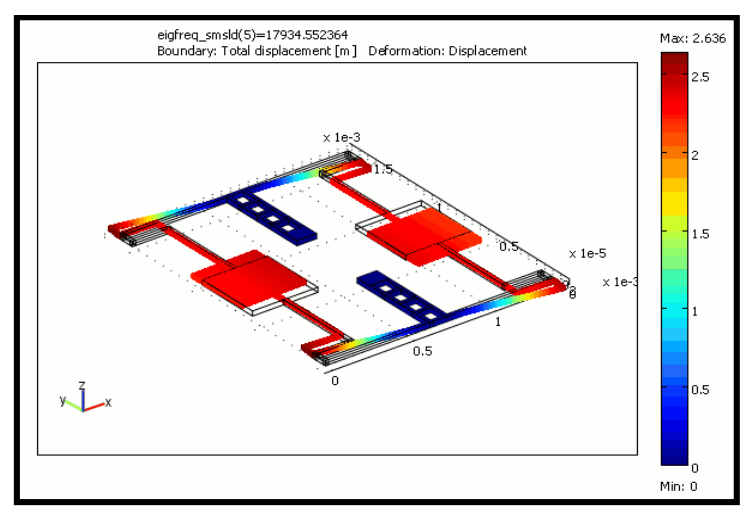

Fig 4: Sense Mode Frequencies (motion along y-axis)

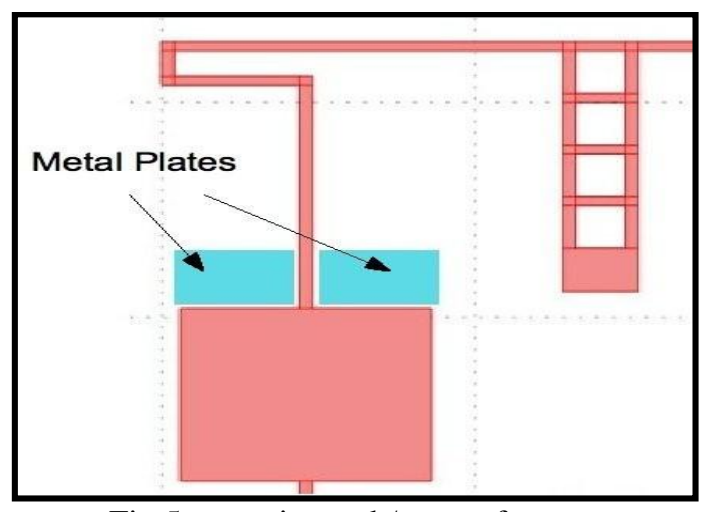

Fig 5: capacitance b/w proof mass and metal Plates

Metal plates are placed above the proof mass as shown in Figure 5 together with the proof mass, these plates form a capacitor. As the proof mass vibrates in drive mode, the distance between the proof mass and the plates remains constant. Since capacitance for a parallel plate capacitor such as this one is a function of the distance between the two plates, in Drive Mode the capacitance also remains constant. Once the structure begins to rotate and enters Sense Mode, as the proof mass moves vertically, the distance between proof mass and plate changes, which change the capacitance. This chance in capacitance can be detected by electronic equipment and converted to indicate the corresponding rotation.

To find the total capacitance of the Comb rive-Proof Mass capacitor, simply multiply the capacity for one finger by the number of fingers, $n$.

The energy stored in the capacitor, $E$ is given as:

$$
\mathrm{E}=\frac{1}{2} \mathrm{CV}^{2}
$$

Where $\mathrm{V}$ is the voltage drop across the capacitor, substituting the value for the total capacitance, $\mathrm{C}$ in equation 1 then energy is given as:

$$
\mathbf{E}=\mathbf{n} \in \in_{0} \frac{l_{0} h}{g} \mathbf{V}^{2}
$$

As the proof mass moves a small distance $\mathrm{x}$ in drive mode, the overlap between fingers changes from $1_{0}$ to $1_{0}+\mathrm{x}$ and energy becomes:

$$
\mathbf{E}=\mathbf{n} \in_{0} \frac{\left(l_{0}+x\right) h}{g} \mathbf{V}^{2}
$$

To find the force $\mathrm{F}$, acting on the proof mass, take the derivative of $\mathrm{E}$ with respect to $\mathrm{x}$ :

$$
\mathbf{F}=\frac{\mathrm{dE}}{\mathrm{dx}}=\mathbf{n} \in \in_{0} \frac{h}{g} \mathrm{~V}^{2}
$$

In order to keep the proof masses in constant motion, an $\mathrm{AC}$ voltages our ce must be used so that the capacitor will not reach a steady state. An AC voltage source is applied to the comb drive transducer as shown below in figure 6 Note that a DC voltage source is applied to the anchor of the gyroscope for use in sense mode. 


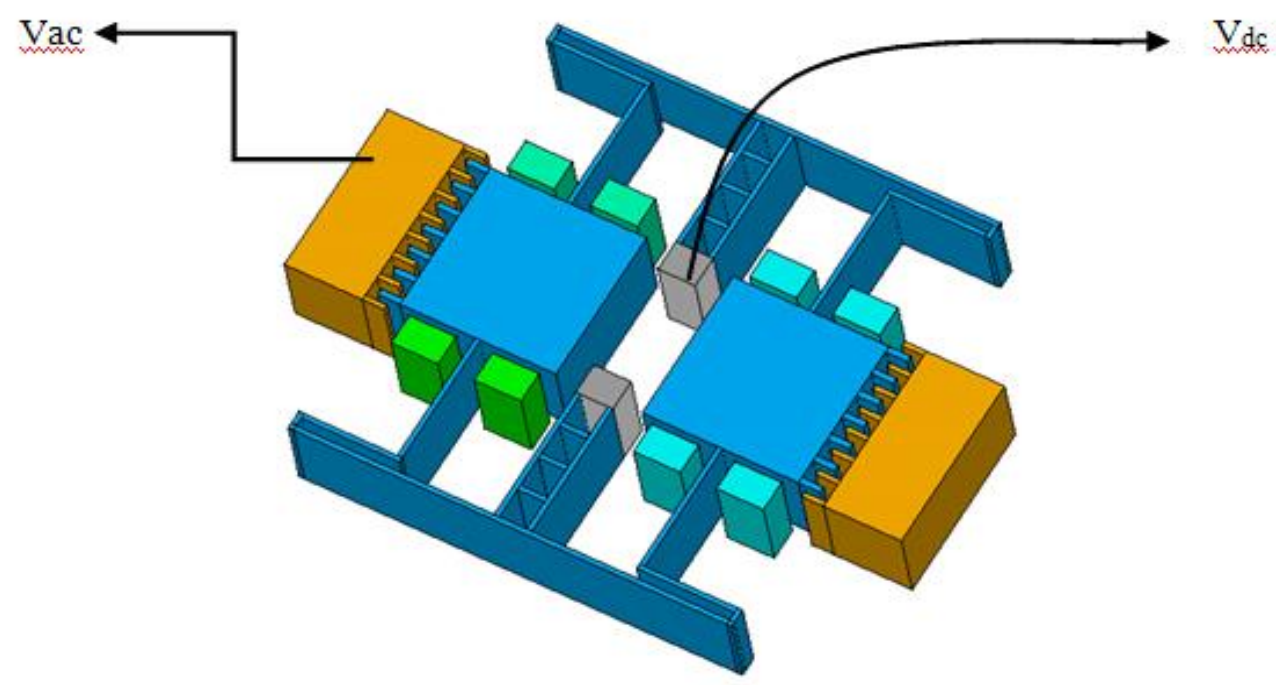

Fig 6: Voltage Sources for Drive Mode

With the set voltage sources applied, the equation for force becomes:

$$
\mathbf{F}=\mathbf{n} \varepsilon_{0} \frac{h}{g}\left(V_{d c}+V_{a c}\right)^{2}
$$

This can be expanded as:

$$
\mathbf{F}=\mathbf{n} \varepsilon_{0} \frac{h}{g} V_{d c}^{2}+\mathbf{n} \varepsilon_{0} \frac{h}{g} V_{a c}^{2}+2 \mathbf{n} \varepsilon_{0} \frac{h}{g} V_{d c} V_{a c}
$$

The term $\mathbf{n} \varepsilon_{0} \frac{\boldsymbol{h}}{\boldsymbol{g}} \boldsymbol{V}_{\boldsymbol{d} \boldsymbol{c}}^{2}$ is a constant and will only offset the proof mass initially by a small amount. Because it will not contribute to the oscillation of the proof mass, it can be disregarded.

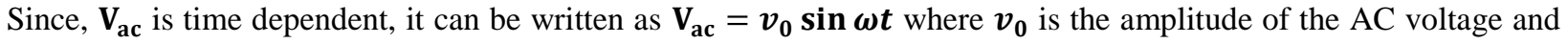
$\boldsymbol{\omega}$ is the angular frequency of the $\mathrm{AC}$ voltage, which must be equal to the drive mode natural frequency of the gyroscope.

By neglecting some of the minor values the equation 6 of force become:

$$
\mathrm{F}=2 \mathrm{n} \varepsilon_{0} \frac{h}{g} V_{d c} v_{0} \sin \omega t
$$

By applying some of the differential calculation the displacement of the proof mass can be calculated as

$$
\begin{aligned}
\boldsymbol{q}_{\mathbf{0}} & =\frac{F}{\boldsymbol{k}} \boldsymbol{Q} \\
= & 2 \mathrm{n} \varepsilon_{0} \frac{h}{g k} V_{d c} v_{0} Q
\end{aligned}
$$

Where $\mathrm{Q}$ is a large number typically greater than 10,000 , and $\mathrm{k}$ is the spring constant for the system.

This equation 8 gives the amplitude of the proof mass vibration in terms of the amplitude of the AC voltage, which can be used to design the comb drive transducer to achieve specific amplitude of proof mass vibration.

\section{Monitoring Drive Mode}

As mentioned above, only one of the comb drive transducers is required to apply a force to the proof mass to keep it in motion. This leaves the other proof mass free to be used to monitor the vibration of the proof mass in drive mode. 
By measuring the current $\mathbf{i}$ from this transducer, the displacement of the proof mass can be found through the following relationship.

Since Q, the electric charge, can be expressed as CV, and current is merely the derivative of charge with respect to time; an expression for electric current can be obtained by evaluating

$$
\frac{d Q}{d t}=\frac{d\left(C \times V_{d c}\right)}{d t}
$$

By applying chain rule of differentiation and putting $\frac{d V_{d c}}{d x}=0$, it results as

$$
i=\frac{d C}{d t} V_{d c}
$$

From equation 2, the derivative of $\mathrm{C}$ with respect to time with a small displacement of $\mathrm{x}$ is given as

$$
\frac{d C}{d t}=\frac{2 n \varepsilon_{0} h}{g} \frac{d x}{d t}
$$

Where $x=q_{0} e^{i \omega t}$ and its differentiation is equal to $\left(\mathrm{i}_{\omega \mathrm{x}}\right)$, by putting all these values along with equation 11 , the value of $i$ in equation (10) become:

$$
i=\frac{2 n \varepsilon_{0} h}{g} \boldsymbol{i} \boldsymbol{\omega} \boldsymbol{x}\left(V_{d c}\right)
$$

\section{Electrical Theory of Sense Mode}

Just as in drive mode a comb drive transducer is used to indirectly measure the proof mass vibration, in sense mode, capacitance is also used to track the vibration of the proof mass. However, sense mode does not use a single comb drive transducer, but rather a pair of metal plates which form a pair of parallel plate capacitors with the proof mass. Both plates over each proof mass are connected to the detection equipment, which helps to amplify their signal in order to aid detection.

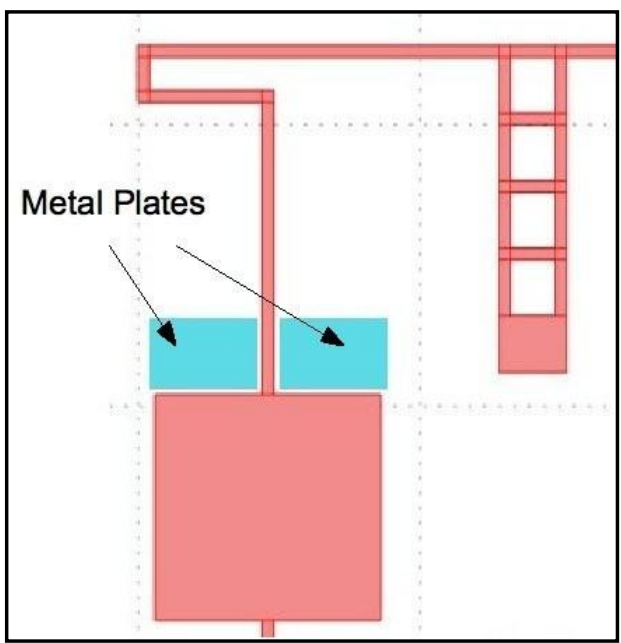

Fig 7: Sense Mode Parallel Plate Capacitor

The initial capacitance for a parallel plate capacitor is given as $C_{0}=\frac{\varepsilon_{0} w h}{d_{0}}$, where $\mathrm{w}$ and $\mathrm{h}$ are the width and height of the plate, respectively, $d_{0}$ and is the gap between the plates. Given that the sense-mode current can be expressed as

$$
i_{s}=\frac{c_{0}}{d_{0}} \frac{d y}{d t} V_{d c}
$$


And that $\frac{d y}{d t}=\omega_{s} y_{0}$ where $\omega_{s}$ is the sense mode angular frequency and $y_{0}$ is the amplitude of the sense mode vibration. The amplitude of sense mode vibration is a function of the force applied to the proof mass:

$$
y_{0}=\frac{F \times Q_{s}}{\left(k-k_{\text {elec }}\right)}
$$

Where $Q_{s}$ is a coefficient relating to the damping of the system, just as in drive mode, $\mathrm{k}$ is the stiffness constant of the system, and $k_{\text {elec }}$ is the Electrostatic Stiffness of the system, which is defined as $k_{\text {elec }}=C_{0} \frac{V_{d c}^{2}}{d_{0}^{2}}$. Since the force acting on the proof masses in sense mode is a result of the Coriolis acceleration, it can be expressed as:

$$
F=m\left(2 \Omega_{z} \times V_{d}\right)
$$

Where $\mathrm{m}$ is the mass of the gyroscope, $\Omega_{z}$ is the rotation signal input to the system and $V_{d}$ is the drive mode velocity of the proof masses, which can be expressed as the product of the drive mode vibration amplitude and the drive mode angular velocity, $q_{0} \omega_{d}$. As a scalar, the force can be expressed as

$$
F=2 m \Omega_{z} q_{0} \omega_{0}
$$

By substituting value of force from equation 16 to equation 14 then to equation 15 , the value of $i_{s}$ become

$$
i_{S}=\frac{C_{0} V_{d c} \omega_{s} 2 m \Omega_{z} q_{0} \omega_{d} Q_{s}}{d_{0}\left(k-k_{\text {elec }}\right)}
$$

The sensitivity of the gyroscope, $\frac{i_{s}}{\Omega_{z}}$ then becomes,

$$
\frac{\mathrm{i}_{\mathrm{s}}}{\Omega_{\mathrm{z}}}=\frac{C_{0} V_{d c} \omega_{s} 2 m q_{0} \omega_{d} Q_{s}}{d_{0}\left(k-k_{\text {elec }}\right)}
$$

\section{SIMULATION OF SCIENCE SPACE VEHICLE}

As an application of Gyroscope and mechanical design, my team and I was honored by a Tenderfoot Award in an annual CanSat competition organized by NASA, USA. This award is for the best and unique design of our "Science Vehicle". The use of MEMS Gyroscope in this Science Vehicle is to stabilize the image of the ground from a height of nearly $800 \mathrm{~m}$. This was achieved with the help of 3 -servo motors which are attached to each other. Another antigyro mechanism was used to control the speed of the Science Vehicle with the help of blades which were tilted in such a way that they were able to reduce the speed of Science Vehicle.

The purpose was to simulate the Science Vehicle so that it could travel through a planetary atmosphere sampling the atmospheric composition like outside pressure, temperature, velocity, decent rate etc. during descent. Following are the detail objectives which were taken care of:

- To carry the hen's egg intact for the entire duration from launch to landing.

- To control the descent rate of the Science Vehicle's Payload Between $4 \mathrm{~m} / \mathrm{s}$ and $10 \mathrm{~m} / \mathrm{s}$ By using Auto Gyro Recovery method/ Passive Helicopter Recovery Method

- To stabilize the Science Vehicle's payload at minimum altitude of 300 meters.

- To Record a Video in the nadir (Earthing Point) direction with the camera on the Science Vehicle's Payload.

- To stabilize the video camera such that the image of the ground shall maintain one orientation with no more than $+/-90$ degree rotation.

- The payload shall collect telemetry data during descent and send it to a central ground station. 
- To Plot the data in real time during flight on ground station.

- To deploy the Payload from container at the optimum altitude after deployment from rocket.

- To use a three-axis accelerometer to measure the stability and angle of descent of the Science Vehicle's Payload during descent.

The whole competition was processed in following three parts, pre-launch, during launch and post launch.

The following Figure 8, shows Launch and Descent Operations of the Science Vehicle during the competition.

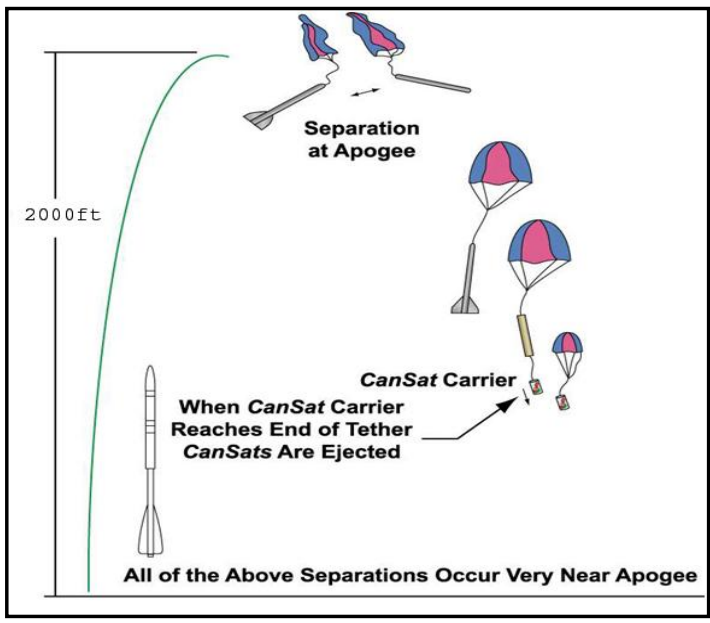

Fig 8: Launch and Descent Operations

The operation is started with a launch of the Science Vehicle by putting it into a demo rocket and launched it to height of nearly $1500 \mathrm{~m}$ above the ground. At the achieved height the rocket dispatched the Science Vehicle in the atmosphere, where it is free to move under its own weight and its own mechanism. With the help of various mechanisms the decent rate of both payload and container were controlled during decency.

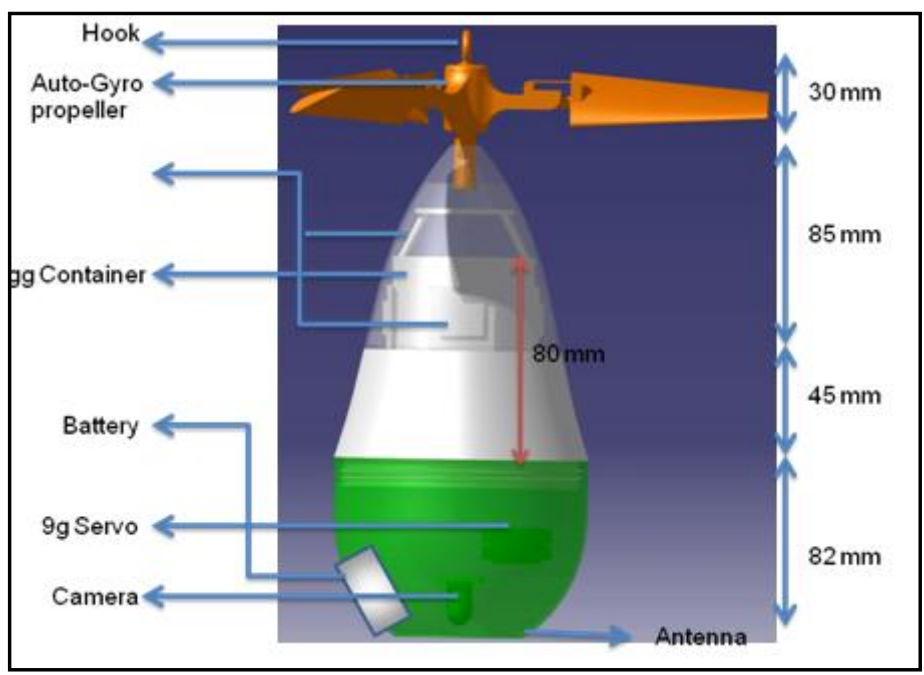

Fig 9: Mechanism of Auto Launch

\section{Image Stabilization Concept}

The setup is made for the image stabilization while descending from the apogee. This includes various parts which are listed as follow: 
$>9 \mathrm{~g}$ servo with rotation

$>9 \mathrm{~g}$ servo with rotation

$>$ Camera module with auto recording in video mode

$>$ Camera and servo holders

$>$ Wires for the connection of camera with controller

Image stabilizing platform consists of platform which is balanced by movement of three servo motors in opposite direction to the movement of the platform. Atmega 328 process the tilt angles obtained from IMU and give instruction to the respective servo motors to rotate by certain angle depending on its previous position to balance or control the platform. IMU consists of ITG3200 Gyroscope which gives 3 DOF. Three servo motors are used for giving stabilization in roll pitch and yaw axis, yaw axis control is given by $360^{\circ}$ motor and two $180^{\circ}$ servo for pitch and roll as shown in the figure 10 below:

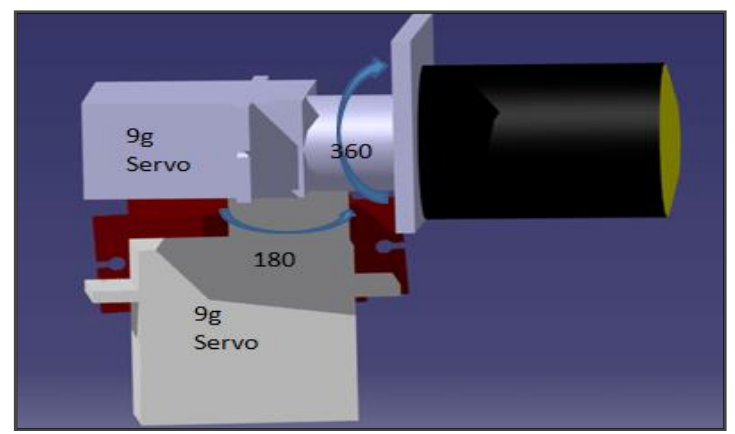

Fig 10: Servo Assembly

\section{Communication and Data handling process}

Communication and Data Handling (CDH) sub-system is responsible for communication between CanSat and ground station. Its main purpose is transmitting required information in stated format from CanSat to ground station. Communications between the CanSat and the base station will be carried out via Xbee transceivers

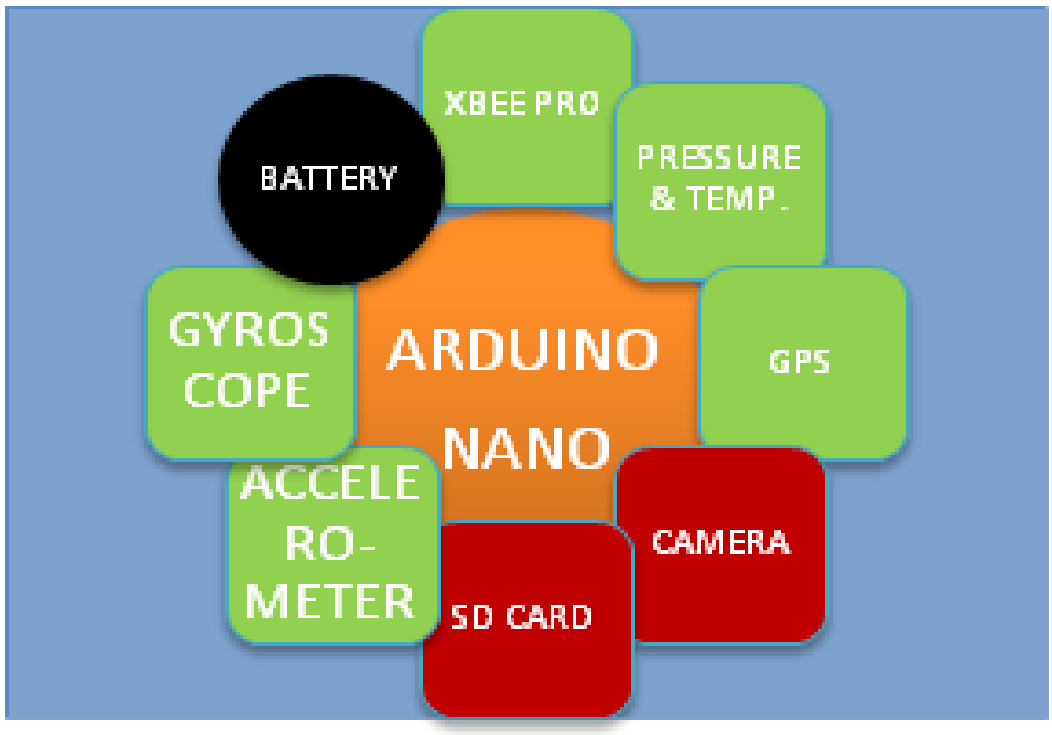

Fig 11: Communication System Overview

Various devices are connected to the microcontroller, out of these one of the main components is gyroscope for the measurement of angular shift of the camera while travelling to the ground. 


\section{Connections of camera assembly with gyroscope}

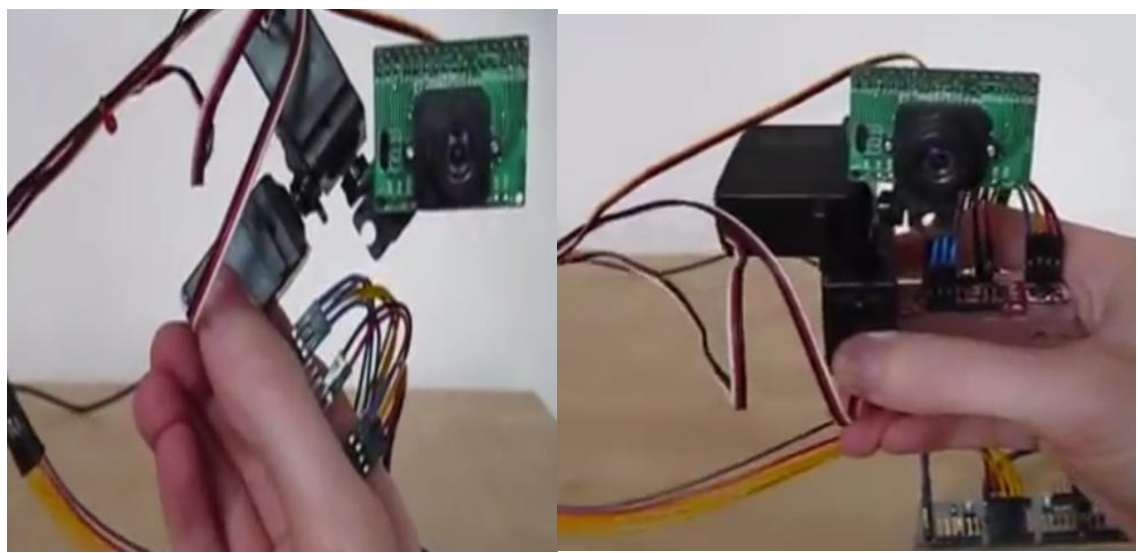

Fig 12: Camera Connections

The camera is connected to the gyroscope with the help of the microcontroller. The process for controlling the camera module is as follow,

No. of pulses required for 1 rotation $=\mathrm{n}$

Angle of rotation in single rotation is $=$

No. of pulse require for of rotation $\theta=$

This angle is divided into two parts

Clockwise angular shift $=$

Anti clockwise angular shift $=$

Angular shift calculated in various directions with the help of gyroscope is first stored in the memory of controller and by comparing this shift with $\theta$ then a unique signal of positive or negative pulses is transferred to the camera assembly attached to the controller.

The telemetry operation is carried out in every 1 second of the flight. The angular change is measured in terms of positive or negative data digits with 8 bit frame.

\section{CONCLUSION}

Image stabilization is achieved during the competition with the help of the gyroscope. Image is stored in the memory card attached to the breakout board, and the card is given to the NASA team members for the inspection and to generate the results. By collecting the information of gyroscope module, various types of the application will become a great achievement .One gyroscope; it is estimated to control other devices containing gyroscopes with the help of microcontrollers and RF modules. The change in one device will allow change in other attached device. These devices are attached with the help of RF modules.

\section{REFERENCES}

[1] Ali Esmaeili, Mehrnaz Aghanouri Kupaeia, Hamed Faghihiana, Hamid Reza Mirdamadia, "An adaptable broadband MEMS vibratory gyroscope by simultaneous optimization of robustness and sensitivity parameters" pp. 132-137, 2013

[2] Tao Jiang, Anlin Wang, Jiwei Jiao, Guangjun Liu "Detection capacitance analysis method for tuning fork micromachined gyroscope based on elastic body model" pp. 52-59, 2006

[3] Wei Wang, Xiaoyong Lv, Dingjie Xu "Design of multi-degree-of-freedom micromachined vibratory gyroscope with double sense-modes" pp. 6-11, 2014

[4] Sang Won Yoona, Sangwoo Lee, Khalil Najafi "Vibration-induced errors in MEMS tuning fork gyroscopes" pp. 32-44, 2012

[5] Arashk Norouzpour-Shirazi, Mohammad Faisal Zaman and Farrokh Ayazi “A Digital Phase Demodulation Technique for Resonant MEMS Gyroscopes” pp. 3260-3266, IEEE SENSORS JOURNAL, VOL. 14, NO. 9, SEPTEMBER 2014 
[6] Hung Ngoc Vu, Nhat Sinh Ha, Long Quang Nguyen, Dzung Viet Dao and Hoang Manh Chu "Design and Analysis of an Integrated 3-DOF Sensor for Tracking in-Plane Motion" pp. 68-72, 2013 IEEE

[7] Vithya Saahar, Raja Durai "DESIGNING MEMS BASED TUNING FORK GYROSCOPE FOR NAVIGATION PURPOSE” pp. 11021107, International conference on Communication and Signal Processing, April 3-5, 2013

[8] Sheng-Ren Chiu, Chung-Yang Sue, Chih-Hsiou Lin, Shih-Ting Lin, Shih-Chieh Lin, Yu-Wen Hsu1 and Yan-Kuin Su "Design, Fabrication and Performance Characterizations of an Integrated Dual-Axis Tuning Fork Gyroscope" 2012 IEEE

[9] Heewon Jeong, Kiyoko Yamanaka, Yasushi Goto and Takanori Aono "Three-Axis MEMS Inertial Sensor for Automobile Applications" 2011 IEEE

[10] Chun-Wei Tsai, Kai-hsin Chen, Ching-Kai Shen, and Jui-che Tsai “A MEMS Doubly Decoupled Gyroscope With Wide Driving Frequency Range” pp. 4921-4929, IEEE TRANSACTIONS ON INDUSTRIAL ELECTRONICS, VOL. 59, NO. 12, DECEMBER 2012

[11] Xuguo Huang, Ping Sun, Zhi Wang “3-DOF MEMS Gyroscope Modeling and Design” pp. 849-852, International Conference on Electronic \& Mechanical Engineering and Information Technology, 2011

[12] Xuezhong Wu, Liqiang Xie, Jianchun Xing, Peitao Dong, Haoxu Wang, and Jianbin Su "A Z Axis Quartz Tuning Fork Micromachined Gyroscope Based on Shear Stress Detection” pp. 1246-1252, IEEE SENSORS JOURNAL, VOL. 12, NO. 5, MAY 2012

[13] Thakur Praveen Singh, Koji Sugano, Toshiyuki Tsuchiya and Osamu Tabata "Analysis of Acceleration Sensitivity in MEMS Tuning Fork Gyroscope" pp. 2006-2009, 2011 IEEE

[14] Wen Yongpeng, Shang Huilin "Modeling and Simulation for a Vibratory Tuning-fork MEMS Gyroscope" pp. 605-608, Third International Conference on Measuring Technology and Mechatronics Automation, 2011

[15] Alexander A. Trusov, Igor P. Prikhodko, Sergei A. Zotov and Andrei M. Shkel "Low Dissipation Silicon Tuning Fork Gyroscopes for Rate and Whole Angle Measurements" pp. 2763-2770, IEEE SENSORS JOURNAL, VOL. 11, NO. 11, NOVEMBER 2011

[16] Zhong Yang Guo, Long Tao Lin, Qian Cheng Zhao, Zhen Chuan Yang, Huikai Xie and Gui Zhen Yan "A Lateral-Axis Microelectromechanical Tuning-Fork Gyroscope With Decoupled Comb Drive Operating at Atmospheric Pressure" pp. 458-468, Journal Of Microelectromechanical Systems, VOL. 19, NO. 3, JUNE 2010

[17] Z. Y. Guo, Z. C. Yang, L. T. Lin, Q. C. Zhao, J. Cui, X. Z. Chi, and G. Z. Yan "Decoupled Comb Capacitors for Microelectromechanical Tuning-Fork Gyroscopes" pp. 26-28, IEEE Electron Device Letters, VOL. 31, NO. 1, JANUARY 2010.

[18] Alexander A. Trusov, Adam R. Schofield, and Andrei M. Shkel "Study of Substrate Energy Dissipation Mechanism in in-Phase and AntiPhase Micromachined Vibratory Gyroscopes" pp. 168-171, 2008 IEEE

[19] Mohammad Faisal Zaman, Ajit Sharma, Zhili Hao and Farrokh Ayazi "A Mode-Matched Silicon-Yaw Tuning-Fork Gyroscope with Subdegree-Per-Hour Allan Deviation Bias Instability” pp. 1526-1536, Journal of Microelectromechanical Systems, VOL. 17, NO. 6, DECEMBER 2008

[20] Adam R. Schofield, Alexander A. Trusov, and Andrei M. Shkel "Multi-Degree of Freedom Tuning Fork Gyroscope Demonstrating Shock Rejection" pp. 120-123, 2007 IEEE

[21] Marc S. Weinberg, Member, ASME, and Anthony Kourepenis "Error Sources in In-Plane Silicon Tuning-Fork MEMS Gyroscopes" pp. 479-491, JOURNAL OF MICROELECTROMECHANICAL SYSTEMS, VOL. 15, NO. 3, JUNE 2006

[22] M.F. Zaman, A. Sharma, and F. Ayazi "High Performance Matched-Mode Tuning Fork Gyroscope" pp. 66-69, 2006 IEEE

[23] Andrei M. Shkel "Type I and Type II Micromachined Vibratory Gyroscopes" pp. 586-593, 2006 IEEE

[24] Ajit Sharma, Faisal M. Zaman, Babak V. Amini and Farrokh Ayazi “A High-Q In-Plane SOI Tuning Fork Gyroscope” pp. 467-470, 2004 IEEE

[25] Damrongrit Piyabongkarn and Rajesh Rajamani "The development of a MEMS gyroscope for absolute angle measurement" pp. 185-195, IEEE TRANSACTIONS ON CONTROL SYSTEMS TECHNOLOGY, VOL. 13, NO. 2, MARCH 2005

[26] Byeung-Leu1 Lee, Sang-Woo Lee, Kyu-Dong Jung, Joon-Hyock Choi, Taek-Ryong Chung, and Yong-Chul Cho "A De-coupled vibratory gyroscope using a mixed micro-machining technology" International Conference on Robotics \& Automation Seoul, Korea, May 21-26, pp. 3412-3416, 2001

[27] S. Sassen, R. Voss, J. Schalk, E. Stenzel, T. Gleissner, R. Gruenberger, F. Neubauer, W. Ficker, W. Kupke, K. Bauer, M. Rose “Tuning fork silicon angular rate sensor with enhanced performance for automotive applications" pp. 80-84, 1999

[28] M. Yachi, H. Ishikawa and Y. Satoh "Design Methodology of Single Crystal Tuning Fork Gyroscope for Automotive Applications" pp. 463-466, 1998 IEEE

[29] Subaru Kudo, Sumio Sugawara and Noboru Wakatuki "Finite element analysis of single crystal tuning forks for gyroscope” pp. 640-647, 1996 IEEE

[30] F. Paoletti, M.A. Grdtillat and N.F. de Rooij “A silicon micromachined tuning fork gyroscope” pp. 3/1-3/6, 1996 IEEE

[31] Defense Advanced Research Projects Agency (DARPA),http://www.darpa.mil/MTO/

[32] Kovacs, G.T.A., Micromachined Transducers Sourcebook, McGraw-Hill, New York,NY, 1998.

[33] Lion, K.S., Transducers: Problems and Prospects, IEEE Transactions onIndustrial Electronics and Control Instrumentation, Vol. IECI-16, No.1, July 1969, pp.2-5.

[34] White, R.M., A Sensor Classification Scheme, IEEE Transactions on Ultrasonics,FerroelectricsAndFrequencyControl,VolUFFC34,No.2,March1987,Pp.124-126.

[35] Ristic,Lj.[Ed.],Sensor Technology And Devices, Artech House, London,1994. 